\section{Translating mRNA vaccines}

\section{By Chris Cain, Senior Writer}

A collaboration between CureVac GmbH and the Friedrich Loeffler Institute has produced in vivo evidence that an mRNA-based vaccine can prevent influenza A infection. ${ }^{1}$ The results provide proof of concept for the company's vaccine platform in infectious disease, and Sanofi has options to the technology.

Conventional methods for influenza vaccine production rely on culturing the virus in chicken eggs or more recently in mammalian cells, followed by antigen purification. However, these approaches have multiple limitations, including a risk of contamination and an inability to rapidly upscale supply in response to an outbreak of a new strain., ${ }^{2,3}$

To overcome these limitations and simplify production, groups have sought to engineer nucleic acid-based influenza vaccines. However, DNA-based vaccines have proven to be poorly or inconsistently immunogenic in humans. ${ }^{4}$

CureVac CEO Ingmar Hoerr told SciBX that this presented an opportunity for the company's mRNA-vaccine platform, which has induced immune responses in clinical trials of the company's therapeutic cancer vaccines. "In a prostate cancer Phase I/IIa study we saw T cell and antibody responses to our mRNA vaccine," he said.

In 2008, CureVac began collaborating with Lothar Stitz, director of the Institute of Immunology and professor of virology and immunology at the Loeffler Institute, to design and test mRNA-based vaccines to prevent influenza.

Hoerr said that the collaboration brought CureVac infectious disease expertise it did not have in-house. "Our strength is in the technology itself, in mRNA and chemical formulations, and also in understanding the immune response. But we needed expertise in animal challenge models, and, by collaborating with academics, we were able to gain access to that. In addition, it was important to show that another group could use our mRNA approach and get the same results-it is proof of concept that we can distribute the technology."

CureVac worked with Stitz's group to design and test mRNA vaccines encoding the influenza $A$ virus hemagglutinin (HA), influenza A virus neuraminidase (NA) and influenza A virus nucleoprotein (NP) antigens. The vaccines incorporated the company's RNActive design technology, which includes optimizing mRNA base-pair content, engineering untranslated regions (UTRs) and complexing with

protamine, an arginine-rich protein that binds and stabilizes mRNA.

In mice, vaccination with a single dose of mRNA encoding both HA and NA antigens led to complete protection against strain-matched influenza infection, whereas mice injected with control antigen died.

In ferrets, which more closely model human infection, injection with an mRNA-encoded HA vaccine induced an antibody titer that would meet requirements for licensure under EMA guidelines. In pigs, injection of a vaccine containing HA, NA and NP prevented disease, whereas unvaccinated pigs showed mild to moderate signs of infection.

Josef Thalhamer, professor of allergy and immunology at the University of Salzburg, told SciBX that the data make a compelling case for further exploration of the mRNA vaccine. "The data are strong and clearly indicate the induction of protective immunity after vaccination with an mRNA vaccine. In particular, the results of the immunizations in pigs can be considered a breakthrough. They demonstrate that a moderate dose of an mRNA vaccine can trigger protective antibody responses against infectious diseases in large animals, which suggests this vaccine type may be effective in humans as well."

Results were published in Nature Biotechnology.

\title{
Broad application
}

The mRNA vaccine could offer a host of potential manufacturing and efficacy advantages over conventional flu vaccines.

The researchers demonstrated that the mRNA vaccine could be stored for 3 weeks at $37^{\circ} \mathrm{C}$ without losing efficacy. Stitz added that production of clinical-grade vaccine could be achieved within 6-8 weeks of the identification of influenza antigen sequence.

Methods for rapid influenza vaccine production are sorely needed. In 2009, the World Health Organization said it would take about five to six months for the first supplies of approved vaccine to become available once a new strain of influenza virus with pandemic potential is identified and isolated.

The new product also could offer longer-term, broader protection against influenza than traditional vaccines.

"The problem with the conventional vaccines is that the virus needs to be inactivated, and dead vaccines only induce a strong neutralizing B cell response but no T cell response," noted Stitz. "The nice thing about this approach is that mRNA actually mimics an infection, as the respective protein coded for in the mRNA is synthesized within the cell, so the antigen can both induce antibodies and enter the MHC antigen presentation pathway for T cells."

"The differential kinetics of antigen exposure between RNA- and proteinbased vaccines could lead to more potent and durable immune responses." -Christian Mandl, Novartis AG
Stitz's team demonstrated this response by injecting mice with an mRNA vaccine encoding NP, which provided T cell-dependent protection from infection with a heterologous influenza strain.

Christian Mandl, VP and global head of virology at Novartis AG's Vaccines \& Diagnostics Inc. division, agreed that a broad, prolonged immune response is a key potential advantage for RNA vaccines.

"Expression of antigen in situ by nucleic acid vaccines, including RNA, has the potential to provide prolonged antigenic stimulus in contrast to 
protein-based vaccines, which are injected as a bolus and typically cleared rapidly," he said. "The differential kinetics of antigen exposure between RNA- and protein-based vaccines could lead to more potent and durable immune responses."

He added, "Preclinical work on RNA prophylactic and therapeutic vaccines has demonstrated their ability to elicit potent and broad immune responses, including functional antibodies, Th1-type T cell responses and cytotoxic T cells. Hence, RNA vaccines have the potential as a platform technology to address a wide variety of infectious diseases caused by viruses, bacteria and parasites, as well as noninfectious diseases such as cancer."

Novartis did not disclose the status of its RNA vaccine programs, but earlier this year Mandl's team published an extensive review on the topic and also described the development of a self-replicating RNA vaccine that protected mice from respiratory syncytial virus (RSV) infection. ${ }^{5,6}$

Gary Nabel, who stepped down this month as director of the Vaccine Research Center at the NIH to become SVP and CSO at Sanofi, told SciBX he was impressed with the progress on RNA vaccines. "It's really pretty remarkable that the RNA technology is working. I think it's a very promising development. We all think of RNA as being incredibly unstable, so it's pretty mindboggling that you could do this with mRNA. The advances have come from stabilizing the RNA and engineering it to ensure it is efficiently translated."

Nabel wants to see results in humans. "The contributions of T cells to flu vaccine efficacy are more theoretical at this point than proven. There are reasons to think it's a good thing, but most animal studies show that a good antibody response is the main component you need for protection. The bottom line is that the jury is still out on what it really can do, and until you test it in people, you don't know what it can do."

\section{Next steps}

CureVac is now collaborating with Sanofi's Pasteur vaccines division, InCell-Art S.A.S. and the U.S. Department of Defense's Defense Advanced Research Projects Agency (DARPA) to develop mRNA vaccines against undisclosed pathogens through the RN Armor Vax consortium.

The consortium was established last November with a total budget of $\$ 33.1$ million. For new technology developed under the project, DARPA will receive a nonexclusive license for U.S. government use, which excludes commercial purposes. Sanofi has commercial options to use CureVac's RNActive technology to develop vaccines for predefined pathogens.

CureVac is eligible for up to $€ 150.5$ million ( $\$ 206.6$ million) per pathogen in upfront and milestone payments from Sanofi, plus tiered royalties. In-Cell-Art, a French company that specializes in macro- molecular delivery technology, will provide Sanofi with undisclosed formulation technologies for mRNA-based vaccines and is eligible for undisclosed upfront payments, milestones and royalties.

Hoerr did not disclose whether influenza was among the predefined pathogens included in the consortium but said it was a logical pathogen to select for proof-of-concept studies because of the wealth of existing preclinical data available for comparison.

He told SciBX that CureVac is still focused on its clinical-stage oncology programs but sees the development of vaccines for infectious disease as a logical additional avenue for the company to pursue. The company's lead prostate cancer program, CV9103, is a four-antigen, mRNA-encoded vaccine that is in Phase IIb to treat metastatic, castration-resistant prostate cancer (mCRPC). Results are expected in 2016.

Stitz said he plans to continue to collaborate with the company on potential mRNA vaccine candidates, and the group has unpublished data for the approach against rabies virus. He said his group also plans to explore the basic biology questions underlying the approach. These include further characterizing mRNA uptake by cells and determining where and through which signaling pathways the vaccine is acting to stimulate an immune response.

Cain, C. SciBX 5(49); doi:10.1038/scibx.2012.1273

Published online Dec. 20, 2012

\section{REFERENCES}

1. Petsch, B. et al. Nat. Biotechnol.; published online Nov. 25, 2012; doi:10.1038/nbt.2436

Contact: Lothar Stitz, Friedrich Loeffler Institute, Greifswald-Insel Riems, Germany

e-mail: lothar.stitz@fli.bund.de

Contact: Karl-Josef Kallen, CureVac GmbH, Tuebingen, Germany e-mail: josef.kallen@curevac.com

2. Usdin, S. \& McCallister, E. BioCentury 17(20), A1; May 4, 2009

3. Ulmer, J.B. et al. Nat. Biotechnol. 24, 1377-1383 (2006)

4. Kutzler, M.A. \& Weiner, D.B. Nat. Rev. Genet. 9, 776-788 (2008)

5. Geall, A.J. et al. Proc. Natl. Acad. Sci. USA 109, 14604-14609 (2012)

6. Ulmer, J.B. et al. Vaccine 30, 4414-4418 (2012)

\section{COMPANIES AND INSTITUTIONS MENTIONED}

CureVac GmbH, Tuebingen, Germany

Friedrich Loeffler Institute, Greifswald-Insel Riems, Germany

In-Cell-Art S.A.S., Nantes, France

National Institutes of Health, Bethesda, Md.

Novartis AG (NYSE:NVS; SIX:NOVN), Basel, Switzerland

Sanofi (Euronext:SAN; NYSE:SNY), Paris, France

University of Salzburg, Salzburg, Austria

U.S. Department of Defense, Washington, D.C.

World Health Organization, Geneva, Switzerland 